\title{
Sistema automatizado de análisis de movimiento para la detección del factor de riesgo ergonómico en la industria de la construcción
}

\author{
Jaime A. León-Duarte, Guillermo Martínez-Cadena, y Jaime Olea-Miranda \\ Universidad de Sonora, Departamento de Ingeniería Industrial, Blvd. Luis Encinas J, 83000, Hermosillo, Sonora. México. \\ (correo-e: jaime.leon@unison.mx; guillermo.martinez.cadena@gmail.com; Jaime.olea@unison.mx)
}

Recibido Jun. 9, 2021; Aceptado Jul. 29, 2021; Versión final Ago. 26, 2021, Publicado Dic. 2021

\begin{abstract}
Resumen:
En este estudio se propone un sistema automatizado para evaluación ergonómica, a partir del método OWAS, en un proceso de doblado de barras de acero en una empresa constructora de México. El sistema evalúa posturas y movimientos de operadores del área de producción utilizando el sensor Kinect (Microsoft). Para realizar la evaluación ergonómica, el software procesa la información que captura el dispositivo mediante la posición de las articulaciones del cuerpo. Se especifica la creación, desarrollo y prueba del sistema en un proceso repetitivo que, historicamanete, provoca el mayor número de lesiones e incapacidades laborales. Se obtiene como resultado una herramienta automatizada capaz de desarrollar evaluaciones ergonómicas en tiempo real. Se demuestra estadísticamente $(95 \%$ de confianza) la confiabilidad y efectividad del sistema comparando la evaluación automatizada del software y una evaluación tradicional de un experto. Se concluye que el sistema automatizado diseñado proporciona evaluaciones ergonómicas con resultados confiables y seguros que pueden servir para una buena toma de decisiones.
\end{abstract}

Palabras clave: ergonomía; evaluación; método OWAS; Kinect; software

\section{Automated motion analysis system for detecting ergonomic risk factor in the construction industry}

\begin{abstract}
:
The present study aims to develop an automated ergonomic evaluation system, based on the OWAS method, for assessing a steel bar bending process performed at a construction company in Mexico. The system evaluates production operators' postures and movements by using Microsoft's Kinect sensor. To perform each ergonomic evaluation, the software processes information captured by the sensor, which detects movement of human body joints. The proposed methodology specifies the creation, development, and testing of the system during a repetitive operation, which is known historically for causing the highest number of injuries and work-related disabilities. The result is an automated tool capable of developing ergonomic evaluations in real time. Reliability and effectiveness is demonstrated statistically ( $95 \%$ confidence) by comparing the software's automated evaluation with that of an expert (traditional evaluation). It is concluded that the automated system developed here delivers ergonomic evaluations that yield reliable and safe results that can improve decisionmaking processes.
\end{abstract}




\section{INTRODUCCIÓN}

La ergonomía es la ciencia que busca la adaptación entre el trabajador, la maquinaria, las herramientas y el ambiente físico de la jornada laboral, con la finalidad de adecuar los puestos de trabajo a las características físicas y psicológicas del trabajador (Venegas y Cochachin, 2019). El principal objetivo de la ergonomía es prevenir daños en la salud del individuo considerando sus tres dimensiones: física, mental y social. Los principios ergonómicos buscan adecuar y adaptar los sistemas de trabajo a las capacidades de las personas que interactúan con ellos para así evitar alteraciones en la salud provocadas por el mal diseño de las actividades (Molina et al., 2019). Diariamente, un número excesivo de personas muere en el mundo a consecuencia de una enfermedad o un accidente relacionado con el trabajo. Se estima que al año se presentan 2.78 millones de muertes a consecuencia del trabajo, de las cuales el $86.33 \%$ están relacionados con una enfermedad laboral. La organización internacional del trabajo (OIT) estima que anualmente se presentan alrededor de 374 millones de lesiones laborales, lo que conduce a una pérdida de días laborables muy cercana al $4 \%$ del PIB mundial (OIT, 2019).

La industria de la construcción representa uno de los principales motores de la economía del mundo, esto, debido a la demanda e inversión en los bienes y servicios para mejorar la calidad de vida y por el desarrollo de la infraestructura para el crecimiento del entorno (Montaña, 2020). De acuerdo a Bedoya et al. (2018), un entorno laboral que mantiene expuesto al trabajador a condiciones de riesgo representa un costo futuro para las organizaciones; por tal motivo es necesario garantizar la salud de los empleados monitoreando las actividades que se desarrollan en el área de trabajo.Gran parte de las lesiones laborales se originan por una carga excesiva de trabajo durante la jornada laboral de los operadores y por un pobre diseño del área de trabajo. Yovi (2020), señala que la fatiga muscular depende de varios factores: la carga de trabajo tanto estática como dinámica, los factores psíquicos y orgánicos del operador y el entorno laboral en el cual desarrolla sus actividades. Conforme la fatiga laboral se incrementa, aparecen las contracturas, el dolor y finalmente, la lesión. Las investigaciones enfocadas al estudio de lesiones causadas por movimientos repetitivos, han manifestado la existencia de factores que intervienen en la aparición de las lesiones musculoesqueléticas. Las lesiones relacionadas a las actividades repetitivas generalmente se presentan en los tendones, los músculos, los nervios del hombro, el antebrazo, la muñeca y la mano. Los diagnósticos médicos para estos tipos de lesiones suelen ser muy variados: tendinitis, peritendinitis, tenosinovitis, mialgias y atrapamientos de nervios distales. Hoffman et al. (2017), señalan que en la actualidad los trastornos músculoesqueléticos son una de las principales causas de enfermedad laboral en el trabajo; además, los transtornos músculo-esqueléticos no solo afectan la calidad de vida en el trabajador, sino que importante costo social y económico.

Las evaluaciones ergonómicas de los puestos de trabajo se utilizan para determinar el nivel de riesgo al que se exponen el trabajador en su entorno laboral. El evaluar un puesto de trabajo, requiere de la aplicación de varios métodos de evaluación ergonómica, debido a que, por cada tarea que el operador realiza existen diversos riesgos presentes (Hoffman et al., 2017). Podemos encontrar una gran variedad de métodos de evaluación ergonómica, sin embargo, los más utilizados para determinar el factor de riesgo de las tareas que realizan los operadores son: método Job Strain Index (JSI), método Rapid Upper Limb Assessment (RULA), la ecuación NIOSH, el método de Evaluación Postural Rápida (EPR), el método Ovako Working Analysis System (OWAS). De igual manera existen métodos de análisis integral del entorno de trabajo, como el método del Laboratorio de Economía y Sociología del Trabajo (LEST), como lo señala Llorca et al., (2015).

JSI es un método fácil de aplicar, se utiliza para determinar el riesgo a sufrir un trastorno músculo-esquelético en las extremidades superiores del cuerpo humano, su principal limitación es la subjetividad en la que puede caer el evaluador si no cuenta con la debida capacitación para desarrollar la evaluación. A diferencia de JSI, el método RULA permite calcular el factor de riesgo de una manera más precisa, pues toma en cuenta las extremidades superiores e inferiores. Desafortunadamente, el desarrollo del método RULA requiere de un nivel de capacitación más alto, el evaluador puede llegar a caer en la subjetividad debido a que se trata de una metodología que analiza un gran número de elementos. La ecuación de NIOSH es una técnica que permite determinar el peso adecuado que debe cargar el individuo, a diferencia del resto de los métodos, la ecuación de NIOSH solo especifica el peso a cargar, más no determina el factor de riesgo al que se enfrenta el operador. Por otra parte, los métodos LEST y EPR son 2 técnicas de evaluación general, mientras que el primero toma en cuenta factores psicosociales y de carga mental a través de un cuestionario extenso y tedioso para evaluar las condiciones del puesto de trabajo, el segundo evalúa la jornada de trabajo del operador para solo determinar si es necesario aplicar un método de evaluación especifico.

Otro método muy utilizado es el método OWAS, dicha evaluación consiste en evaluar las extremidades superiores e inferiores del cuerpo mientras el operador realiza sus actividades laborales, esto, con la finalidad de identificar todas aquellas posturas inadecuadas que ponen en riesgo la integridad física del operador, desafortunadamente, si no se cuenta con el nivel de capacitación necesario para desarrollar la evaluación, es 
muy común caer en la subjetividad, provocando evaluaciones erróneas (Llorca et al., 2015). Un resumen de las características generales de estos métodos aparece en la tabla 1.

Tabla 1: Comparativa entre los métodos de evaluación ergonómica (Adaptada de Llorca et al., 2015).

\begin{tabular}{|c|c|c|c|}
\hline Método & Objetivo & Tipo de método & $\begin{array}{l}\text { Segmento corporal } \\
\text { evaluado }\end{array}$ \\
\hline JSI & $\begin{array}{l}\text { Identificar actividades propensas a ocasionar } \\
\text { trastornos musculo esqueléticos en las } \\
\text { extremidades superiores (Llorca et al., 2015). }\end{array}$ & $\begin{array}{l}\text { Evaluación de } \\
\text { tareas repetitivas }\end{array}$ & Extremidades superiores \\
\hline RULA & $\begin{array}{l}\text { Evaluar la exposición de los trabajadores a } \\
\text { factores de riesgo que pueden ocasionar } \\
\text { trastornos músculo-esqueléticos en los miembros } \\
\text { superiores del cuerpo (Llorca et al., 2015). }\end{array}$ & $\begin{array}{l}\text { Evaluación de } \\
\text { postura }\end{array}$ & $\begin{array}{l}\text { Extremidades superiores e } \\
\text { inferiores }\end{array}$ \\
\hline $\mathrm{NIOSH}$ & $\begin{array}{l}\text { Definir el levantamiento ideal bajo las condiciones } \\
\text { necesarias para evitar giros y posiciones } \\
\text { incorrectas en el operador (Llorca et al., 2015). }\end{array}$ & $\begin{array}{l}\text { Evaluación de } \\
\text { carga }\end{array}$ & - \\
\hline LEST & $\begin{array}{l}\text { Evaluar las condiciones del área de trabajo } \\
\text { tomando en cuenta los aspectos psicosociales y la } \\
\text { carga mental (Llorca et al., 2015). }\end{array}$ & Evaluación general & - \\
\hline EPR & $\begin{array}{l}\text { Realizar una evaluación de forma global a lo largo } \\
\text { de la jornada del operador con el fin de determinar } \\
\text { si es necesario aplicar métodos de evaluación más } \\
\text { específicos (Llorca et al., 2015). }\end{array}$ & Evaluación general & - \\
\hline OWAS & $\begin{array}{l}\text { Mejorar los procedimientos de trabajo eliminando } \\
\text { todas aquellas posturas forzadas que se presentan } \\
\text { al realizar la tarea. (Llorca et al., 2015). }\end{array}$ & $\begin{array}{l}\text { Evaluación de } \\
\text { postura }\end{array}$ & $\begin{array}{l}\text { Extremidades superiores e } \\
\text { inferiores }\end{array}$ \\
\hline
\end{tabular}

Mas et al., (2017) enfatizan que estos métodos, aun cuando son altamente utilizados, el nivel de formación de los evaluadores del puesto trabajo no es adecuado, lo que conduce a resultados de evaluaciones ergonómicas poco confiables, debido al factor subjetivo y de apreciación personal agregado por un evaluador con poca experiencia y capacitación. Dicho lo anterior, el objetivo del presente trabajo fue desarrollar un sistema automatizado con la ayuda del dispositivo Kinect para la evaluación ergonómica en tiempo real de los movimientos que realiza el operador al llevar a cabo sus actividades laborales y poder así, prevenir lesiones musculo-esqueléticas derivadas de la repetitividad de las actividades que exige el proceso a los trabajadores del centro de distribución, así como también, demostrar la eficacia y confiabilidad de la herramienta.

\section{OTROS ANTECEDENTES}

Kinect es un dispositivo capaz de permitir la interacción entre el ser humano y el ordenador mediante gestos, movimientos y comandos de voz. Kinect fue desarrollado por Microsoft para ser utilizado en la consola Xbox, y está compuesto por una cámara RGB, un sensor de profundidad y 4 micrófonos. El sensor de profundidad está conformado por un láser y un sensor infrarrojos, lo cual le permite al dispositivo detectar y seguir los movimientos del cuerpo humano (Tölgyessy et al., 2021). El funcionamiento del Kinect es relativamente simple, el dispositivo emite rayos infrarrojos hacia todos los objetos e individuos que se encuentran dentro del área de alcance, para posteriormente calcular las distancias entre ellos a partir del tiempo que tarda la luz infrarroja en llegar hasta los objetos (Bari y Gavrilova, 2019). Debido a la múltiple gama de aplicaciones de esta tecnología, Microsoft ofrece un kit de desarrollo de software (SDK, Software Developer Kit) para construir aplicaciones que integren dinámicamente personas, objetos y el ambiente. El dispositivo lleva a cabo el reconocimiento del cuerpo humano mediante "Skeleton tracking", el cual consiste en identificar puntos clave, correspondientes a las articulaciones, uniéndolos para generar una representación de la morfología humana, como se observa en la figura 1 (Wibowo et al., 2019).

En estudios previos, Manghisi et al., (2017) demostraron que la utilización del dispositivo Kinect para la evaluación ergonómica a partir del método RULA es efectiva al utilizar el sistema K2RULA, una aplicación informática diseñada para evaluar en tiempo real los movimientos de los operadores, y determinar así el factor de riesgo en extremidades superiores al realizar actividades repetitivas. Al comparar los resultados obtenidos por el sistema y los obtenidos con una evaluación tradicional de un experto, se llegó a la conclusión de que K2RULA puede usarse como una herramienta rápida, confiable y de bajo costo para la evaluación del método RULA. 


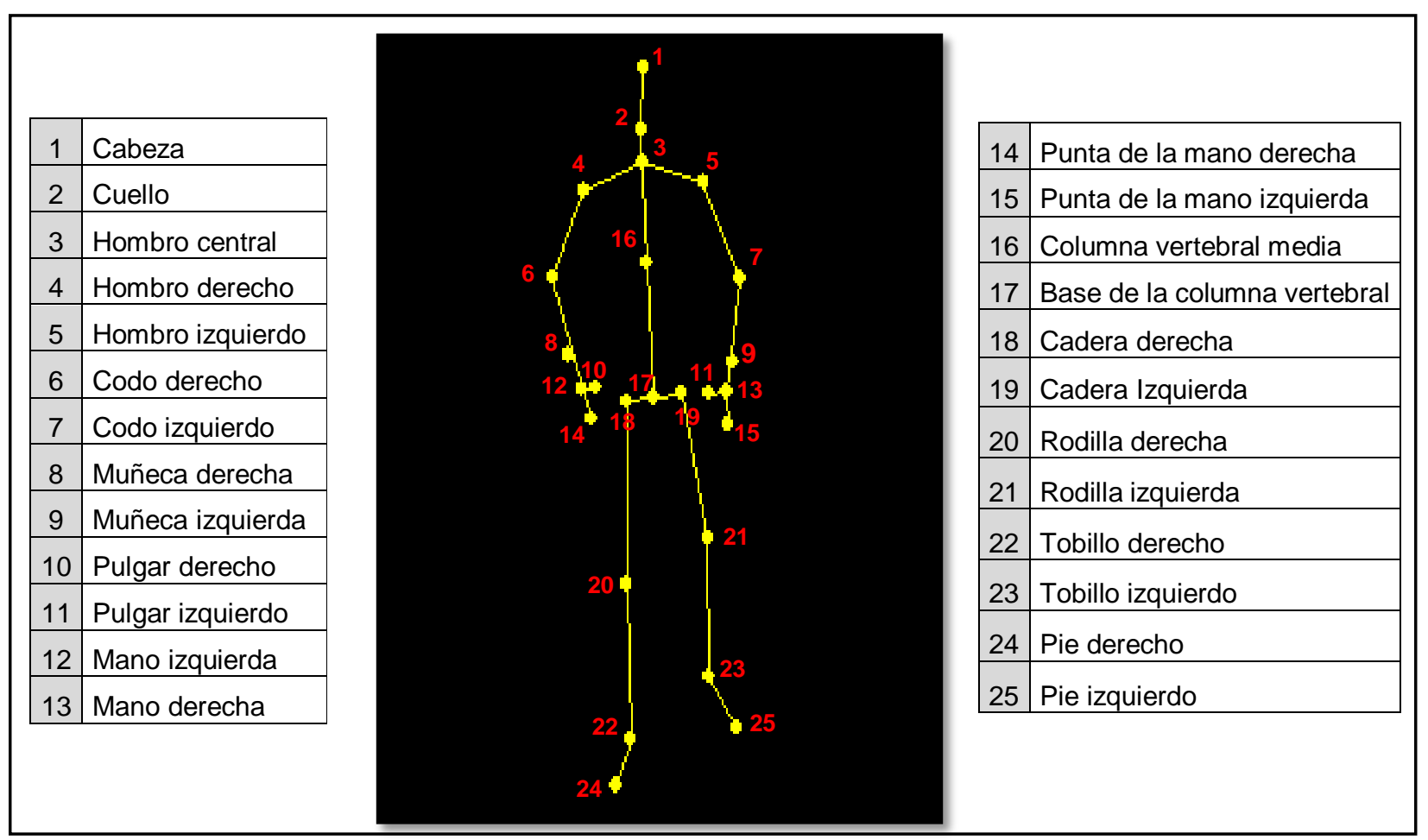

Fig. 1: Articulaciones Skeleton (Adaptada de Wibowo et al., 2019)

Finalmente, Aguilar et al., (2018) desarrollaron un sistema computarizado a partir del método MODAPTS (Organización Modular de Tiempos Normalizados Predeterminados) para calcular el estándar de tiempo de una actividad de trabajo. El proyecto se implementó en una organización dedicada a la fabricación de arneses de cables. Los resultados obtenidos por el software fueron comparados con los resultados obtenidos por un analista humano, pudiendo afirmar estadísticamente que los estándares de tiempo generados por el software son confiables. Gracias a este estudio se logró concluirque un sistema automatizado de captura y análisis de movimiento puede operar sin la necesidad de un supervisor que monitoree su funcionamiento, y que la información recolectada puede ser útil para realizar estudios ergonómicos, especialmente para la detección de posturas peligrosas.

\section{CASO DE APLICACIÓN}

En el centro de distribución de una de las empresas del ramo de la construcción más importantes de México se produce barra de acero doblada de los calibres 3/8" y $1 / 2$ ", para satisfacer la demanda que genera el mercado local y foráneo. Los operadores tienen que levantar desde el suelo 10 barras de acero, si se está trabajando con diámetro de $3 / 8$ ", para colocarlas sobre la maquina dobladora, estas 10 barras representan un peso de $66 \mathrm{~kg}$. Si se va a trabajar con diámetro de 1/2", los operadores tienen que cargar 6 piezas, las cuales pesan $72 \mathrm{~kg}$. en conjunto. Durante una jornada laboral de 8 horas, el proceso de doblado se repite 180 veces si se trabaja con barra de acero de 3/8" y 168 veces si se procesa un diámetro de 1/2". De los años 2018 a 2020 las lesiones músculo-esqueléticas aumentaron un $600 \%$ en el centro de distribución, afectando directamente la producción, que se ha reducido como consecuencia un $9 \%$. Las quejas por dolores en espalda y hombros por parte de los operadores de la estación de doblado de barra de acero se presentan diariamente, lo cual genera preocupación en el departamento de producción La carga de trabajo en obreros y personal supervisor es alta, por lo cual, desarrollar evaluaciones ergonómicas requiere de un tiempo considerable para analizar las actividades que desempeñan los operadores y poder recabar información, por esta razón, en la empresa no se lleva a cabo ninguna evaluación de riesgo, solo se cuenta con un análisis mayormente subjetivo realizado el año 2018 por el supervisor de producción.

Para brindar una alternativa de solución a este problema, a finales del año 2020 se implementó un sistema automatizado, el cual es capaz de realizar evaluaciones ergonómicas en tiempo real a partir del método OWAS y apoyándose de los movimientos del cuerpo humano que son detectados por el sensor Kinect. Las principales propuestas metodológicas corresponden a la planteada por Valdenebro et. al. (2016) y a la propuesta planteada por Muñoz et al., (2013). El modelo propuesto por Valdenebro et. al. (2016) trabaja con la metodología NIOSH y OWAS apoyándose de la fotografía y el video, mientras que la propuesta metodológica de Muñoz et al., (2013) hace uso del sensor Kinect para la medición angular de las principales 
articulaciones del cuerpo, con la finalidad de definir el movimiento y la ubicación del ser humano. Para la implementación del sistema automatizado se siguió una metodología de 4 fases: Análisis del entorno, diseño del sistema, implementación y análisis de resultados, como se aprecia y se describen en la figura 2.

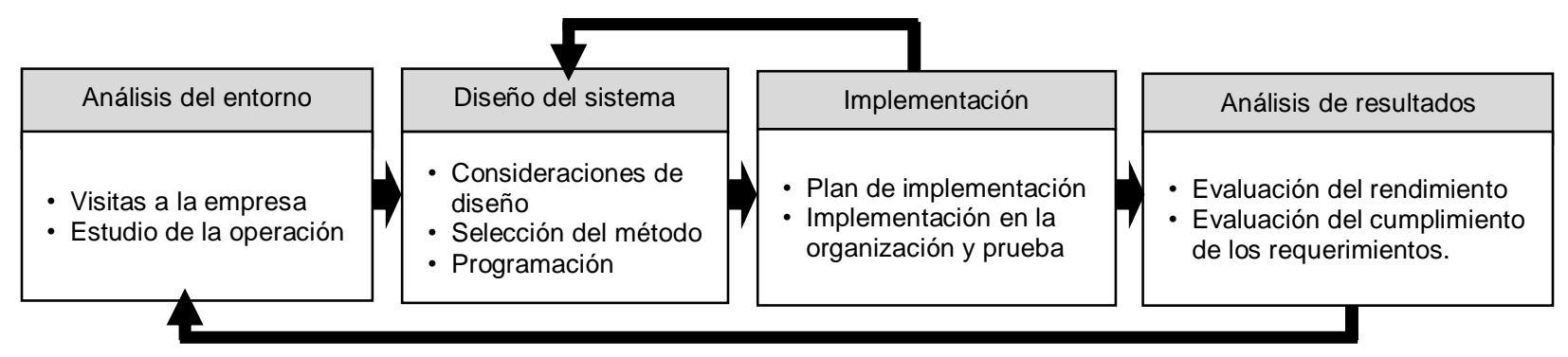

Fig. 2: Modelo metodológico (Adaptado de Valdenebro et al, (2016) y Muñoz et al., (2013)

\section{MATERIALES Y MÉTODOS}

Para diseñar el sistema y seleccionar el método de evaluación ergonómica adecuado, se llevaron a cabo varias reuniones con el personal del centro de distribución, en dichas reuniones se analizó de manera detallada la actividad que los operadores desarrollan en su jornada laboral.

\section{Selección del método de evaluación y programación del sistema}

Para seleccionar el método de evaluación ergonómica a utilizar, se realizó un análisis de las lesiones que han sufrido los operadores del departamento de doblado de varilla en los últimos años, obteniendo como resultado que el método más adecuado es OWAS. Para la codificación se utilizó el software LabVIEW y su extensión Makerhub, la cual cuenta con una librería de controles para manipular el sensor Kinect V2 y extraer los datos necesarios para realizar la evaluación. La interfase principal del sistema se muestra en la figura 3.

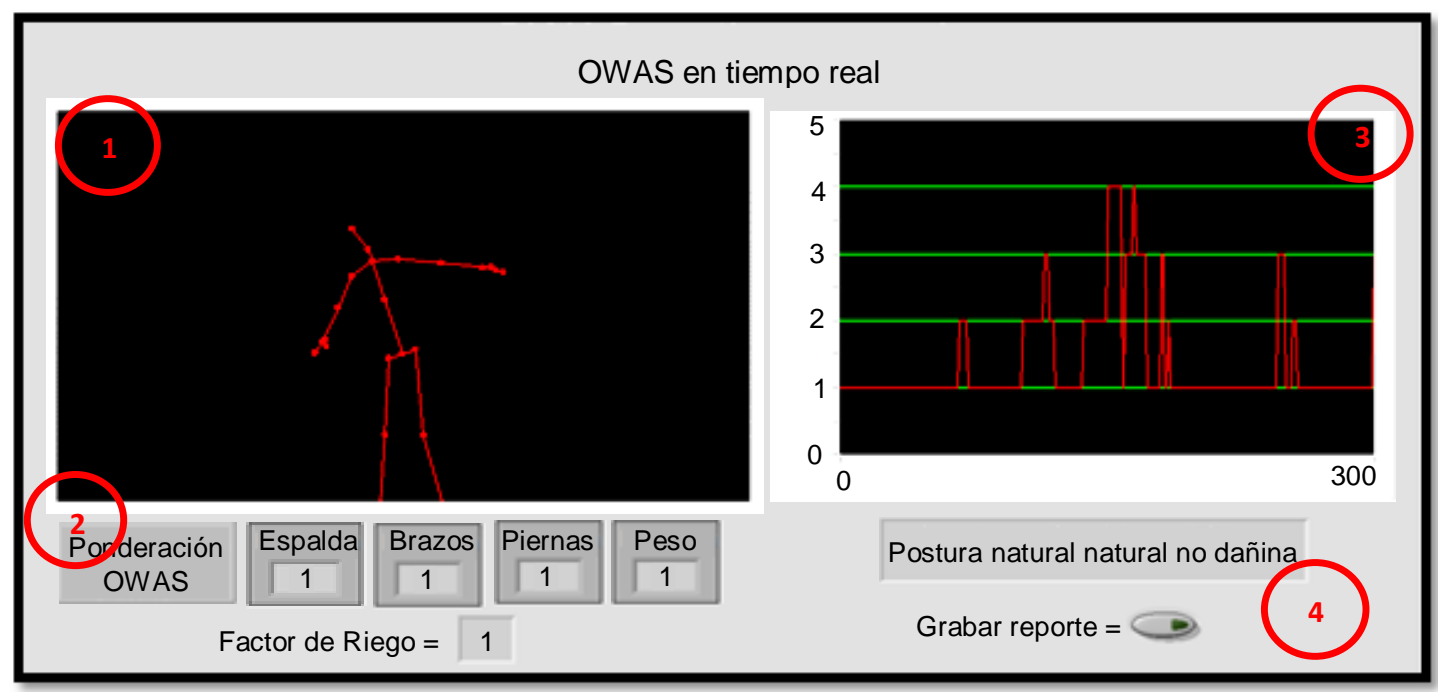

Fig. 3: Pantalla principal del sistema.

1) El visor Skeleton. Es la pantalla que permite visualizar las articulaciones de la persona que ha detectado el sensor Kinect, en esta pantalla se pueden observar los movimientos del individuo que se esta evaluando. 2) Ponderación OWAS y factor de riesgo. En esta parte se muestra la calificación otorgada por el sistema a la espalda, los brazos, las piernas y la carga manipulada por el operador, según la posición adoptada en ese momento. El valor que el método puede otorgar a la espalda va de 1 a 4: se otorga calificación de 1 si la espalda esta recta, 2 si la espalda se encuentra inclinada a más de $20^{\circ}, 3$ si existe una torción superior a $20^{\circ}$ y 4 si existe inclinación y giro al mismo tiempo. En el caso de los brazos el valor que el sistema puede otorgar va de 1 a 3: 1 si ambos brazos se encuentran a una altura menor a la de los hombros, 2 si solo un brazo se encuentra sobre la altura de los hombros y 3 si ambos brazos sobrepasan la altura de los hombros. 
El valor que el sistema otorga a las piernas va del 1 al 7.1 si la persona se encuentra sentada, 2 si la persona se encuentra de pie y con ambas piernas rectas, 3 si la persona tiene una pierna recta y la otra flexionada, 4 si ambas piernas están flexionadas o se encuentra en posición de cuclillas, 5 si ambas piernas están flexionadas o se encuentra en posición de cuclillas y con el peso corporal desequilibrado, 6 si la persona se encuentra de rodillas y 7 si la persona se encuentra caminando. La calificación del peso va de 1 a 3: 1 si el peso de la carga es menor a $10 \mathrm{~kg}$., 2 si el peso de la carga se encuentra entre 10 y $20 \mathrm{~kg}$. y 3 si el peso de la carga sobrepasa los $20 \mathrm{~kg}$. Una vez que el sistema obtiene las 4 calificaciones otorga el factor de riesgo para la posición en cuestión, la ponderación del factor de riesgo va de 1 a 4: 1 si la posición en cuestión no es riesgosa para la salud y no es necesaria una corrección, 2 si se requieren acciones correctivas en un futuro cercano, 3 si se requieren acciones correctivas lo antes posible y 4 si las acciones correctivas se deben realizar de inmediato. 3) Gráfica de control del factor de riesgo. El gráfico de control muestra el historial del factor de riesgo obtenido en los últimos 5 minutos en los que el sistema ha estado registrando la información. 4) Generador de reporte. Al encender este botón la información capturada por el sensor comienza a registrarse en una hoja de cálculo.

\section{Evaluación del sistema}

Antes de iniciar las pruebas del sistema, fue necesario encontrar la posición adecuada que permitiera al sensor Kinect obtener el campo de visión necesario para procesar las imágenes de forma correcta y sin obstrucciones. Para ello, el sensor Kinect fue montado en un tripié y se colocó a una distancia de aproximadamente 2 metros con respecto al operador evaluado. Las pruebas del sistema tuvieron una duración de 3 días en diferentes momentos de la jornada. En cada uno de estos días se realizó una corrida del sistema para extraer la información necesaria. La duración de cada corrida fue de un ciclo de producción. El ciclo de producción inicia cuando el operador se pone de pie frente a la barra de acero y termina cuando coloca las barras sobre la mesa de doblado. Durante cada prueba el supervisor de producción llevaba a cabo la evaluación manual siguiendo el método tradicional, para después comparar sus resultados con los que el sistema arrojó. Cómo referencia, para poder determinar la confiabilidad del sistema se tomaron las 5 posiciones más relevantes del proceso, como se puede observar en la figura 4.

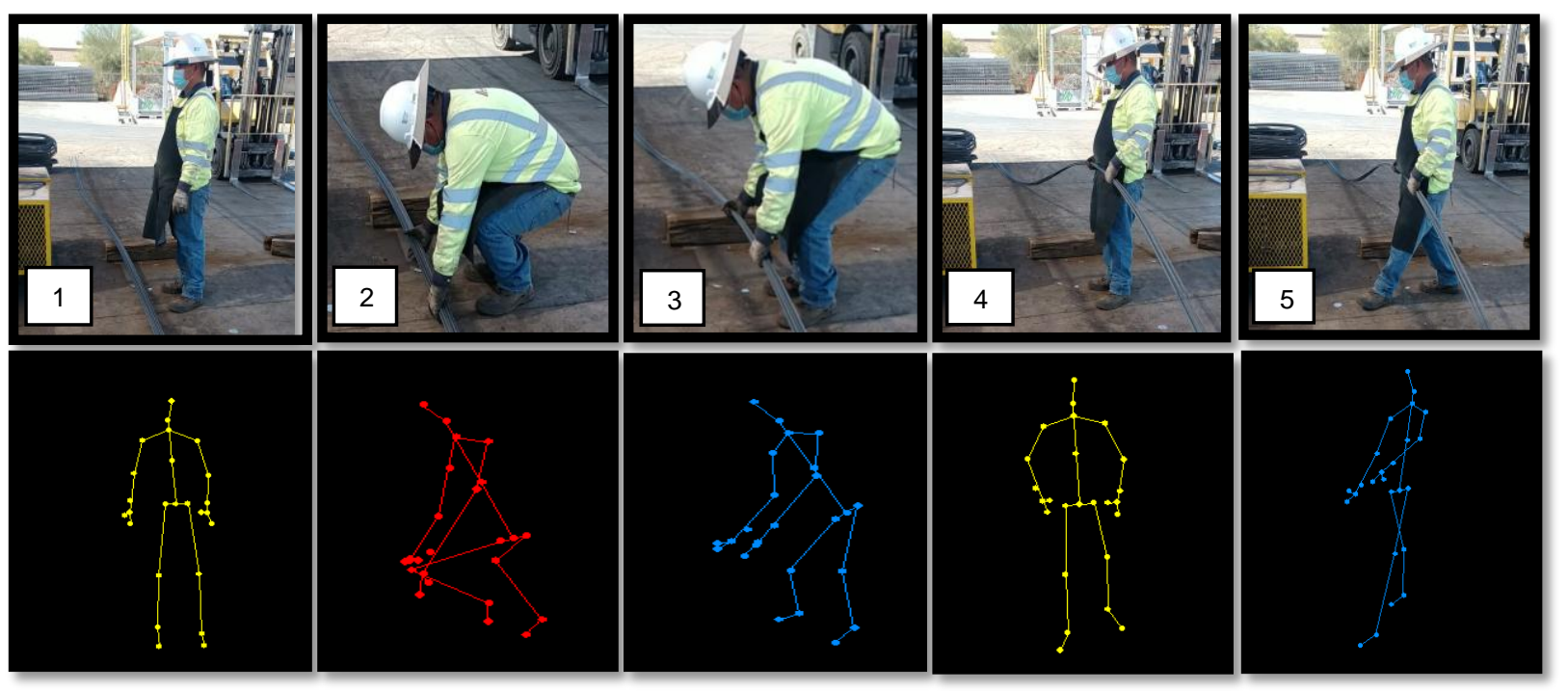

Fig. 4: Principales posiciones durante el proceso de doblado de varilla.

\section{RESULTADOS}

Los resultados que generó el sistema para cada una de las 5 posiciones durante las 3 corridas y los resultados obtenidos por el supervisor al realizar las evaluaciones ergonómicas siguiendo el método tradicional se pueden observar en la tabla 2. Para evaluar la confiabilidad del sistema en base a los resultados obtenidos en las 3 corridas, se aplicó una prueba de hipótesis T para dos muestras de varianzas iguales. La hipótesis nula $(\mathrm{H} 0)$ indicaba que existe desigualdad entre las dos muestras sometidas a estudio, finalmente, el nivel de significancia utilizado en la prueba de hipótesis fue del $5 \%$. El valor de P obtenido en la prueba de hipótesis es de 0.854727826 , mayor a 0.05 lo que indica que no existe suficiente evidencia estadística para decir que existe diferencia en las medias de los datos. Para realizar la prueba de hipótesis se utilizó la extensión de análisis de datos de la sección datos del software Excel, los resultados obtenidos en dicha prueba se observan en la tabla 3. Con esto se puede afirmar que, el realizar una evaluación ergonómica siguiendo la metodología OWAS y utilizando el sistema informático desarrollado, ofrece igual confiabilidad que aplicar la misma evaluación siguiendo el método tradicional. 
Tabla 2: Resultados arrojados por el sistema y siguiendo el método tradicional durante las 3 corridas.

\begin{tabular}{|c|c|c|c|c|c|}
\hline \multirow{4}{*}{ 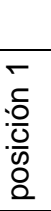 } & \multirow[b]{2}{*}{ F.R. Corrida 1} & \multicolumn{2}{|c|}{ Sistema } & \multicolumn{2}{|c|}{ Método tradicional } \\
\hline & & 1 & Postura normal y sin efectos dañinos. & 1 & Postura normal y sin efectos dañinos. \\
\hline & F.R. Corrida 2 & 1 & Postura normal y sin efectos dañinos. & 1 & Postura normal y sin efectos dañinos. \\
\hline & F.R. Corrida 3 & 1 & Postura normal y sin efectos dañinos. & 1 & Postura normal y sin efectos dañinos. \\
\hline \multirow{3}{*}{$\begin{array}{l}\text { N } \\
\frac{1}{0} \\
: \frac{0}{0} \\
\frac{0}{0} \\
2\end{array}$} & F.R. Corrida 1 & 3 & Postura con efectos dañinos. & 3 & Postura con efectos dañinos. \\
\hline & F.R. Corrida 2 & 3 & Postura con efectos dañinos. & 3 & Postura con efectos dañinos. \\
\hline & F.R. Corrida 3 & 2 & Postura con posibilidad de causar daño. & 3 & Postura con efectos dañinos. \\
\hline \multirow{3}{*}{ 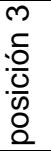 } & F.R. Corrida 1 & 3 & Postura con efectos dañinos. & 3 & Postura con efectos dañinos. \\
\hline & F.R. Corrida 2 & 3 & Postura con efectos dañinos. & 3 & Postura con efectos dañinos. \\
\hline & F.R. Corrida 3 & 3 & Postura con efectos dañinos. & 3 & Postura con efectos dañinos. \\
\hline \multirow{3}{*}{ 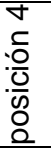 } & F.R. Corrida 1 & 1 & Postura normal y sin efectos dañinos. & 1 & Postura normal y sin efectos dañinos. \\
\hline & F.R. Corrida 2 & 1 & Postura normal y sin efectos dañinos. & 1 & Postura normal y sin efectos dañinos. \\
\hline & F.R. Corrida 3 & 1 & Postura normal y sin efectos dañinos. & 1 & Postura normal y sin efectos dañinos. \\
\hline \multirow{3}{*}{ 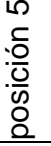 } & F.R. Corrida 1 & 1 & Postura normal y sin efectos dañinos. & 1 & Postura normal y sin efectos dañinos. \\
\hline & F.R. Corrida 2 & 1 & Postura normal y sin efectos dañinos. & 1 & Postura normal y sin efectos dañinos. \\
\hline & F.R. Corrida 3 & 1 & Postura normal y sin efectos dañinos. & 1 & Postura normal y sin efectos dañinos. \\
\hline
\end{tabular}

Tabla 3: Resultados obtenidos al aplicar la prueba de hipótesis.

\begin{tabular}{|l|c|c|}
\hline \multicolumn{3}{|c|}{ Prueba t para dos muestras suponiendo varianzas iguales } \\
\hline & Sistema & Método tradicional \\
\hline Media & 1.733333333 & 1.8 \\
\hline Varianza & 0.923809524 & 1.028571429 \\
\hline Observaciones & 15 & 15 \\
\hline Varianza agrupada & 0.976190476 & \\
\hline Diferencia hipotética de las medias & 0 & \\
\hline Grados de libertad & 28 & \\
\hline Estadístico t & -0.184787287 & \\
\hline $\mathrm{P}(\mathrm{T}<=\mathrm{t})$ una cola & 0.427363913 & \\
\hline Valor crítico de t (una cola) & 1.701130934 & \\
\hline $\mathrm{P}(\mathrm{T}<=\mathrm{t})$ dos colas & 0.854727826 & \\
\hline Valor crítico de t (dos colas) & 2.048407142 & \\
\hline
\end{tabular}

\section{DISCUSION FINAL}

Con la finalidad de encontrar nuevas alternativas tecnológicas que permitan salvaguardar la integridad física de los operadores del centro de distribución se desarrolló una herramienta autónoma y tecnológica capaz de determinar el factor de riesgo al que se enfrentan los operadores con solo detectar la posición de las principales articulaciones de cuerpo. Para el desarrollo del sistema, se utilizó la tecnología del sensor Kinect fabricado por la empresa Microsoft, lo que convierte a esta herramienta en un sistema sobresaliente con respecto a los sistemas estudiados. El aplicar la metodología OWAS con la ayuda del sensor Kinect para determinar el factor de riesgo al que se enfrentan los operadores del departamento de doblado de barras de acero es comparable a la implementación del sistema K2RULA propuesto por Manghisi (2017). Ambos sistemas resultan ser confiables al momento de calcular el factor de riesgo al que se enfrentan los operadores durante la jornada laboral.

Por su parte Muñoz et al., (2013), hacen uso del sensor Kinect de Microsoft para crear una plataforma interactiva conformada por distintos videojuegos que permiten ejercitar el cuerpo humano de pacientes en rehabilitación, mientras que nuestro sistema aprovecha la versatilidad del sensor para realizar el cálculo del grado angular con la finalidad de llevar a cabo un análisis biomecánico que permite identificar el factor de riesgo al cual se enfrenta el operador al realizar sus actividades. Con el sistema implementado en el centro de distribución se lograron alcanzar los objetivos básicos de la aplicación de las evaluaciones ergonómicas, cuya finalidad es detectar aquellas posturas que ponen en riesgo la integridad física de los operadores. El sistema permitirá al jefe del centro de distribución tomar decisiones certeras y tendrá un estudio que servirá como evidencia de respaldo. Por otra parte, el mantener a salvo la integridad física del operador también significa prevenir futuros gastos. Una incapacidad parcial ocasionada por un accidente laboral en el 
departamento de doblado de varilla tiene un costo de $\$ 313.33$ MXN diarios por empleado. Si esta incapacidad resulta ser permanente, el centro de distribución estaría obligado a pagar una pensión (de por vida) que va de los $\$ 234.75$ a los $\$ 313.33 \mathrm{MXN}$ diarios.

\section{CONCLUSIONES}

De acuerdo con los resultados obtenidos y su discusión, se plantean las siguientes conclusiones: 1) El sistema propuesto representa una herramienta informática confiable. Al aplicar la prueba de hipótesis no existe suficiente evidencia estadística para decir que hay diferencias en los resultados arrojados por el sistema y los resultados obtenidos por el método tradicional, por lo cual se puede concluir que el sistema automatizado que se diseñó para el centro de distribución proporciona evaluaciones ergonómicas con resultados confiables y seguros que pueden servir para una buena toma de decisiones. 2) El sistema resultó ser una herramienta muy potente, capaz de proporcionar resultados precisos en fracciones de segundos sin la necesidad de realizar cálculos manuales ni recurrir a la extenuante inspección visual que requiere la aplicación de evaluaciones ergonómicas siguiendo el método tradicional. 3) Otro aspecto muy importante a destacar, es el bajo costo que implica el adquirir el sensor Kinect pues su precio en el mercado oscila entre los US $\$ 100 \mathrm{y}$ US\$150. 4) El utilizar este tipo de sistemas para evaluaciones ergonómicas, evita la subjetividad inherente a la evaluación manual, pues los cálculos se realizan en base a los movimientos y ángulos de las articulaciones captados por un sensor óptico. 5) No es necesaria una capacitación para utilizar la herramienta a diferencia del método tradicional que requiere de una capacitación previa para entender la metodología.

\section{REFERENCIAS}

Aguilar, L., León, J., y Romero, L., A software tool for the calculation of time standards by means of predetermined motion time systems and motion sensing technology, Advances in intelligent systems and computing, $1^{a} \stackrel{\text { ed., }}{1088-1092,}$ Springer, Champagne, Francia (2019)

Bedoya, E., Severiche, C., Sierra, D., y Osorio, I., Work accidents in the construction sector: the case of the district of cartagena de indias (Colombia), Period 2014-2016, http://dx.doi.org/10.4067/S0718-07642018000100020, Información Tecnológica, 29(1), 193-200 (2018)

Bari, H., y Gavrilova, M., Artificial neural network based gait recognition using kinect sensor, https://doi.org/10.1109/ACCESS.2019.2952065, IEEE Access, 7(1), 162708-162722 (2019)

Hoffman K., Parraga, J., y Idrovo, R., Evaluación ergonómica de los puestos de trabajo del área operativa de siembra y empaque, https://doi.org/10.26820/recimundo/1.5.2017.41-51, Mundo de la Investigación y el Conocimiento, 1(5), 41-51 (2017)

Llorca, J., Manual de ergonomía aplicada a la prevención de riesgos laborales, $1^{a}$ ed., 97-105, Ediciones Pirámide, Madrid, España (2015)

Mas, J., Alcaide, J., y Poveda, R., Errors using observational methods for ergonomics assessment in real practice, https://doi.org/10.1177\%2F0018720817723496, Human Factors: The Journal of the Human Factors and Ergonomics Society, 59(1),1173-1187 (2017)

Manghisi, V., Uva, A., y otros 4 autores, Real time RULA assessment using kinect v2 sensor, https://doi.org/10.1016/j.apergo.2017.02.015, Applied Ergonomics, 65(1), 481-491 (2017)

Molina, B., Antón, A., Bravo, D., y Carrasco, M., Ergonomía y calidad de vida laboral en la praxis odontológica, http://dx.doi.org/10.35381/s.v.v3i6.472, Salud y Vida, 3(6) (2019)

Montaña, H., Mozo, V., y Holman, M., Analysis of labor risk indices in the Colombian productive, International Journal of Mechanical and Production Engineering Research and Development (IJMPERD), 10(3), 7715-7722 (2020)

Muñoz, J., Henao, A., y López, J., Sistema de rehabilitación basado en el uso de análisis biomecánico y videojuegos mediante el sensor kinect, http://dx.doi.org/10.22430/22565337.386, TecnoLógicas,1(1), 43-54 (2013)

OIT, Safety and health at work (safety and health at work), https://www.ilo.org/ (2019)

Tölgyessy, M., Dekan, M., Chovanek, L., y Hubinský, P., Evaluation of the azure kinect and its comparison to kinect v1 and kinect v2, https://doi.org/10.3390/s21020413, Sensors, 21(2), 1-23 (2021)

Valdenebro, L., López, M., y otros 3 autores, Evaluación ergonómica de un puesto de trabajo en el sector metalmecánico, Ingeniería Industrial, 1(1), 69-83 (2016)

Venegas, C., y Cochachin, J., Nivel de conocimiento sobre riesgos ergonómicos en relación a síntomas de trastornos músculo esqueléticos en personal sanitario, Rev Asoc Esp Espec Med Trab, 28(1), 126-135 (2019)

Wibowo, J., Alief, N., y Whulanza, Y., Gait analysis using kinect as a home monitoring instrument in case of spine joint movement, https://doi.org/10.1109/QIR.2019.8898273, UI Proceedings on Science and Technology, 2(1), 78-82 (2019)

Yovi, E., Ergonomic analysis of traditional pine oleoresin tapping: musculoskeletal disorders, cumulative fatigue, and job satisfaction, http://dx.doi.org/10.23960/jsl38283-296, Jurnal Sylva Lestari, 8(3), 283-296 (2020) 